(C)2013 American Physical Society. Access to this work was provided by the University of Maryland, Baltimore County (UMBC) ScholarWorks@UMBC digital repository on the Maryland Shared Open Access (MD-SOAR) platform.

Please provide feedback

Please support the ScholarWorks@UMBC repository by emailing scholarworks-group@umbc.edu and telling us

what having access to this work means to you and why it's important to you. Thank you. 


\title{
All-optical-switching demonstration using two-photon absorption and the Zeno effect
}

\author{
S. M. Hendrickson, ${ }^{1}$ C. N. Weiler, ${ }^{1}$ R. M. Camacho, ${ }^{2}$ P. T. Rakich, ${ }^{2}$ A. I. Young, ${ }^{2}$ M. J. Shaw, ${ }^{2}$ T. B. Pittman, ${ }^{3}$ \\ J. D. Franson, ${ }^{3}$ and B. C. Jacobs ${ }^{1}$ \\ ${ }^{1}$ Applied Physics Laboratory, The Johns Hopkins University, Laurel, Maryland 20723, USA \\ ${ }^{2}$ Sandia National Laboratories, Albuquerque, New Mexico 87185, USA \\ ${ }^{3}$ University of Maryland, Baltimore County, Baltimore, Maryland 21250, USA
}

(Received 6 June 2012; revised manuscript received 7 January 2013; published 7 February 2013)

\begin{abstract}
Low-contrast all-optical Zeno switching has been demonstrated in a $\mathrm{Si}_{3} \mathrm{~N}_{4}$ microdisk resonator coupled to warm atomic vapor. The device is based on the suppression of the resonant microcavity field buildup due to nondegenerate two-photon absorption. This experiment used one beam in a resonator and one in free space due to limitations related to device physics. These results suggest that a similar scheme with both beams resonant in the cavity would correspond to input power levels below $150 \mathrm{nW}$.
\end{abstract}

DOI: $10.1103 /$ PhysRevA.87.023808

PACS number(s): 42.65.Pc, 42.82.Et

\section{INTRODUCTION}

There has been significant interest recently in low-power all-optical switches with minimal energy dissipation for applications in such fields as quantum information and classical logic [1-6]. A fundamental challenge to the development of these devices is the limited strength of the nonlinear interaction available within practical design constraints. For example, there have been remarkably successful demonstrations of all-optical switching and related phenomena using trapped atoms [1-4], photonic crystal cavities coupled to quantum dots [5], and novel small mode volume, ultra-high- $Q$ resonators [6], but further development is needed to address issues of operating temperature, bandwidth, and packaging. Fully integrated photonic systems that rely on such effects as the Kerr nonlinearity or free-carrier injection have not yet achieved sufficiently low operating power levels or energy dissipation rates [7-9].

A potential alternative approach to achieving the necessary nonlinear strength is the use of warm atomic vapor with a small mode volume device. Recently there has been significant progress achieving ultralow power nonlinear effects with evanescent microphotonic devices coupled to alkali vapors [10-13]. These devices have demonstrated saturated absorption [10,11], electromagnetically induced transparency [10], modulation [12], and nondegenerate two-photon absorption [13] with low input power levels. Additionally, a number of groups have shown that it is possible to directly integrate atomic vapor cells with photonic waveguides which could be more scalable over other atomic schemes that require full vacuum systems [14-16].

We have previously proposed an all-optical-switching mechanism [17] based on the suppression of the cavity field buildup in an add-drop resonator by nondegenerate two-photon absorption (TPA). This nonlinear dissipation-based switching mechanism is now commonly referred to as the optical analog of the quantum Zeno effect (QZE) [18-20], for reasons we describe below.

In the QZE, frequent measurement can inhibit the unitary evolution of a quantum system [21]. To see how this same effect applies to the four-port resonator system, consider first the classical operation of our device, shown in Fig. 1. With the resonator strongly coupled to two waveguides, the presence of a resonant input at either of the two frequencies will result in the light coupling into the resonator and exiting the opposite waveguide. This is due to the destructive interference between the light remaining in the waveguide and the built-up field amplitude in the cavity that couples back to the waveguide. When both frequencies are present, the TPA prevents the coherent intracavity field buildup and the input beams pass by the resonator because there is insufficient amplitude in the cavity to result in complete destructive interference in the waveguide. Whereas the QZE prevents the buildup of a probability amplitude, the optical analog to the QZE suppresses the coherent buildup of the electromagnetic field amplitude within a microresonator.

To clarify the connection to the quantum Zeno effect, we now consider the same case, but with only a single photon incident into the system and a method to measure whether there is a photon in the cavity. In our system the measurement is performed by atomic absorption but any form of dissipation will do. In the absence of these measurements there will be a probability amplitude buildup within the cavity that results in the photon exiting the drop port, as expected. Next, consider the same case but with the addition of a measurement of whether the photon is in the cavity. If the measurement is performed frequently enough that the amplitude remains small, the most probable result of each measurement will be that no photon is in the cavity and therefore no photon will be absorbed by the atoms. This is the counterintuitive nature of the quantum Zeno effect: By introducing a dissipation channel to the system we are reducing the probability that the photon will ever enter the state where it is subject to such loss. Completing the analogy, since the probability amplitude for the photon to couple into the cavity is suppressed, it will exit in the original waveguide mode, effectively bypassing the cavity altogether, resulting in a switched output.

The potential advantages of Zeno switches have been well documented recently. Huang et al. have predicted that this mechanism can be used to create an interaction-free switching scheme with near-zero signal loss [19]. Wen et al. used inverse Raman scattering (IRS) in a silicon microring resonator to demonstrate that dissipation can be used to achieve excellent channel selectivity such that a single longitudinal cavity mode could be modulated independently [18,22], a 


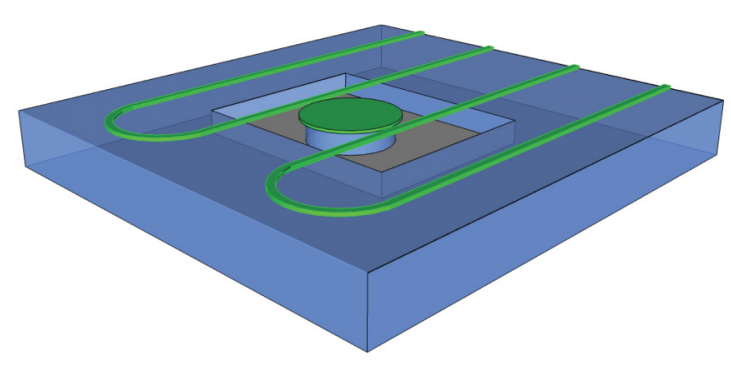

(a)

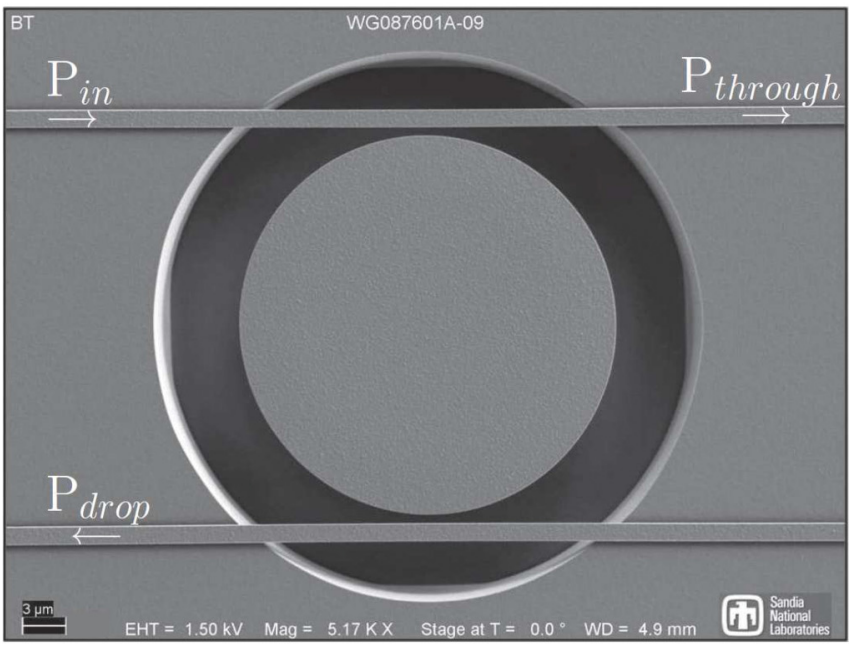

(b)

FIG. 1. (Color online) (a) A schematic showing the chip-level design of the $\mathrm{Si}_{3} \mathrm{~N}_{4}$ microdisk coupled to two waveguides. (b) SEM image of the $\mathrm{Si}_{3} \mathrm{~N}_{4}$ microdisk used in these experiments. Details can be found in the text.

major challenge for systems based on Kerr nonlinearities or free-carrier effects. Kieu et al. demonstrated IRS in a fiber system and demonstrated wavelength selective modulation with approximately $100 \mathrm{~nm}$ of possible operating range, nearly no added insertion loss, and expected bandwidth exceeding $100 \mathrm{THz}$ [20]. Little et al. previously considered electro-optically induced absorption in the context of the add-drop resonator configuration and also noted the potential for ultralow loss switching, especially with the use of small mode volume cavities [23]. Taken together, the literature suggests that Zeno switches may provide capabilities not found in other types of devices. To date, there has not been an all-optical demonstration of Zeno switching that has shown the rerouting of a signal beam using a weakly coupled dissipation mechanism.

Here we present experimental progress towards a Zeno switch consisting of a $\mathrm{Si}_{3} \mathrm{~N}_{4}$ microdisk embedded in warm rubidium $(\mathrm{Rb})$ vapor with clear evidence of signal rerouting. Our approach takes advantage of the narrow atomic resonances and nondegenerate nature of TPA in atomic vapor to show that the presence of one beam can alter the transmission of a second at ultralow power levels. A key aspect of this work is the enhanced rate of TPA that can be achieved at low power levels by confining the light to a small mode volume.

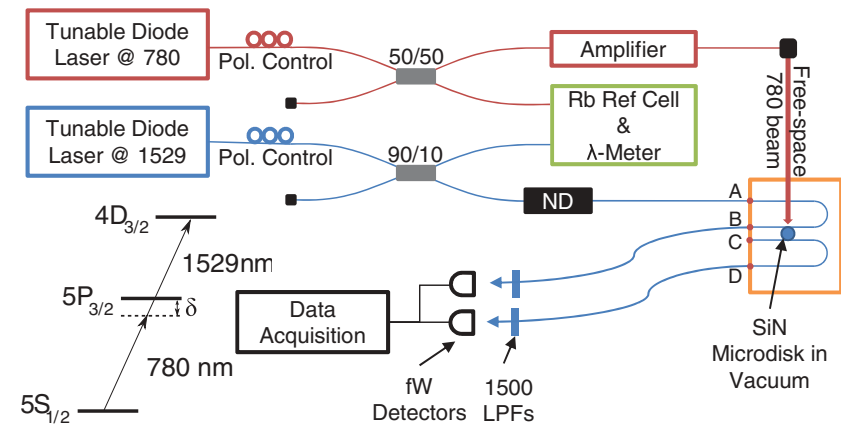

FIG. 2. (Color online) Optical diagram for the experiments described in the text. Not shown is a $25 \mathrm{~mW} 405 \mathrm{~nm}$ laser focused on the resonator for thermal tuning purposes.

\section{EXPERIMENTAL SETUP}

To demonstrate Zeno switching, we used a $\mathrm{Si}_{3} \mathrm{~N}_{4}$ microdisk with a diameter of $26 \mu \mathrm{m}$ and a thickness of $250 \mathrm{~nm}$. These devices were chosen over other high- $Q$ microcavity designs because they have integrated waveguide coupling and a high index of refraction that supports mode compression. The resonators contain no cladding material, allowing the evanescent field to extend outside the device and overlap considerably with the atomic vapor. Each microdisk was critically coupled to two waveguides in an add-drop configuration, and then coupled to four single-mode fibers using a V-groove chip with compatible spacing. A chip-level schematic is shown in Fig. 1(a) and a scanning electron microscope (SEM) image of the waveguide-coupled microdisk is shown in Fig. 1(b).

The setup used in this experiment is shown in Fig. 2. Two frequency-stabilized external-cavity diode lasers were used: one at $780 \mathrm{~nm}$ and one at $1529 \mathrm{~nm}$. Both of these beams passed through fiber splitters and into a reference cell as well as a vacuum system containing a microdisk. The two beams were focused through a Rb reference cell $\left({ }^{87} \mathrm{Rb}\right.$ and $\left.{ }^{85} \mathrm{Rb}\right)$ in a counterpropagating configuration to allow monitoring of the TPA condition while their frequencies were measured using a wavelength meter. The $780 \mathrm{~nm}$ beam passed through a lownoise tapered amplifier prior to entering the vacuum system through a view port where it was focused onto the microdisk. The $1529 \mathrm{~nm}$ beam remained in fiber and was coupled to the resonator via on-chip waveguides. The $1529 \mathrm{~nm}$ through and drop ports were measured using femtowatt detectors after passing through $1500 \mathrm{~nm}$ long-pass filters.

The microdisk was mounted in a vacuum system with the fibers entering through a Teflon feedthrough [24]. The vacuum system was lightly baked to $120{ }^{\circ} \mathrm{C}$ until pressures reached $10^{-8}$ Torr and then cooled to about $100{ }^{\circ} \mathrm{C}$. $\mathrm{Rb}$ vapor was injected into the system using an array of dispensers. The atomic density was estimated using a fit to the tails of the absorption spectrum of a probe beam. This method resulted in an average density between $10^{11}$ and $10^{12} \mathrm{~cm}^{-3}$. The section containing the microdisk was surrounded by view ports to allow optical access from the top, as will be discussed below.

Our scheme uses the two-photon transition $5 S_{1 / 2} \rightarrow$ $5 P_{3 / 2} \rightarrow 4 D_{3 / 2}$ that consists of the well-known $D_{2}$ line near $780 \mathrm{~nm}$ followed by a transition near $1529 \mathrm{~nm}$. Because coupling gaps between the waveguide and the resonator were 
designed for wavelengths near $1529 \mathrm{~nm}$, the $780 \mathrm{~nm}$ transition was excited using a focused free-space beam.

The matching of the cavity resonance $\left(\lambda_{c}\right)$ to the 1529 atomic resonance $\left(\lambda_{\mathrm{Rb}}^{1529}\right)$ was accomplished using a number of steps. Initially, the approximate diameter of the microdisk was chosen to optimize the mode volume according to Ref. [17] with $\lambda_{c}$ near $\lambda_{\mathrm{Rb}}^{1529}$. The diameter was then varied incrementally for a series of disks laid out across each substrate such that a device could be selected after fabrication, coupled to fibers and secured using vacuum-compatible, high-temperature UVcurable epoxy. Once the devices were inserted into the vacuum chamber they experienced a downward shift in $\lambda_{c}$ due to the change in index. It has also been observed that $\mathrm{Si}_{3} \mathrm{~N}_{4}$ devices undergo an upward shift in $\lambda_{c}$ when exposed to $\mathrm{Rb}$ in vacuum as a result of accumulation [25] although this change can be reversed by rinsing the device in distilled water. We have observed that this $\mathrm{Rb}$ shift in $\lambda_{c}$ tends to increase with the maximum vapor density achieved and have used it as a tool to shift the resonance by as much as $7 \mathrm{~nm}$. The resonance can also be shifted down by etching with hydrofluoric acid [25]. Ultimately, each of the aforementioned shifts was compensated for and then final tuning was accomplished thermally with a $25 \mathrm{~mW} 405 \mathrm{~nm}$ laser focused on the resonator.

While it has been predicted that classical Zeno switching could result in excellent signal contrast [17], the quality factor $(Q)$ of this device $\left(\approx 10^{5}\right)$ is currently below what is needed for optimal performance. To best observe transmission changes due to TPA, we designed a data collection scheme to reduce noise through averaging. A calibration algorithm was used to vary the atomic intermediate state detuning $\left(\delta_{\mathrm{Rb}}\right)$ by scanning the frequencies of the 780 and $1529 \mathrm{~nm}$ lasers by an equal and opposite amount such that their total energy remained fixed and resonant with the two-photon transition described previously. This allowed a full scan across the profile of the cavity resonance with the conditions for TPA satisfied, although the strength of the absorption varied as a function of intermediate state detuning. As a control, each resonant TPA scan was followed by a scan with the frequency of the 780 beam offset by $10 \mathrm{GHz}$ such that the cavity resonance was measured in the absence of TPA. To compensate for any thermal drift when averaging data trials, each cavity profile was zeroed to a new frequency axis $(\Delta)$ by fitting to a Lorentzian and then adding a frequency offset $\left(f_{0}\right)$ to the atomic intermediate state detuning $\left(\delta_{\mathrm{Rb}}\right)$,

$$
\Delta=\delta_{\mathrm{Rb}}-f_{0} .
$$

Figure 3 shows the strength of the TPA rate in the reference cell under conditions typical of each trial. As can be seen in the plot, the TPA condition is maintained throughout a scan of approximately $5 \mathrm{GHz}$. The shape of the graph corresponds to the well-known $\delta_{\mathrm{Rb}}^{-2}$ dependence predicted by perturbation theory [17], as shown by the fit.

\section{RESULTS}

An example of Zeno switching is shown in Fig. 4. Approximately 100 trials were averaged for each condition. The blue lines in the upper plot show the cavity drop port (peak) and through port (dip) with the $780 \mathrm{~nm}$ laser detuned so that the conditions for TPA were not satisfied. The red lines

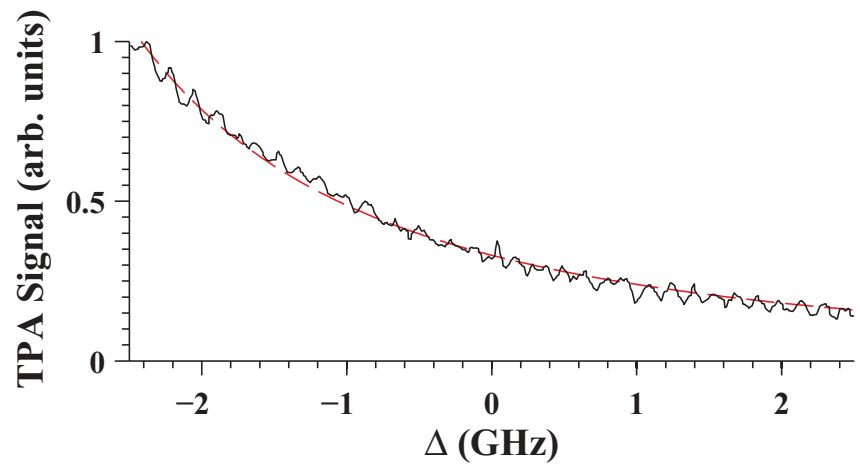

FIG. 3. (Color online) A typical profile of the TPA signal in the reference cell as a function of intermediate state detuning of the $1529 \mathrm{~nm}$ beam. The frequencies of both lasers were scanned simultaneously such that their total energy remained fixed with the ${ }^{85} \mathrm{Rb} F=35 S_{1 / 2} \rightarrow 5 P_{3 / 2} \rightarrow 4 D_{3 / 2}$ transition. The typical cavity position during data collection $(\Delta=0)$ was approximately equal to $f_{0}=6 \mathrm{GHz}$.

in the upper plot show the cavity response in the presence of TPA. The difference in these two situations is highlighted in the lower plot, where it is emphasized that the effect of the additional absorption is to increase the transmission of the through port and decrease the transmission of the drop port, consistent with a low-contrast Zeno switching process.

Figure 5 shows an example of a control case with the only difference being the absence of $\mathrm{Rb}$ vapor. As can be seen in the data, there is no similar change in the transmission of the cavity to suggest a switching event. Although not shown here, additional controls were also performed to validate our data. For example, in one test the cavity was thermally detuned

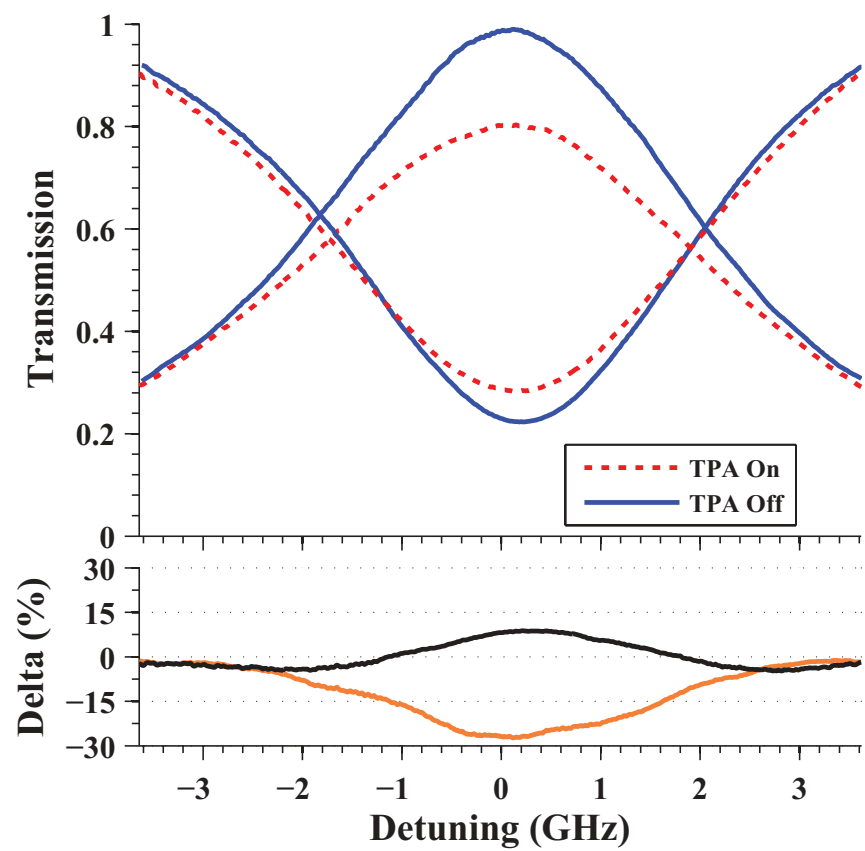

FIG. 4. (Color online) Switching data: The upper plot shows cavity transmission with the conditions for TPA satisfied (dashed) and a control (solid), as described in the text. The lower plot shows the difference in the signals. 


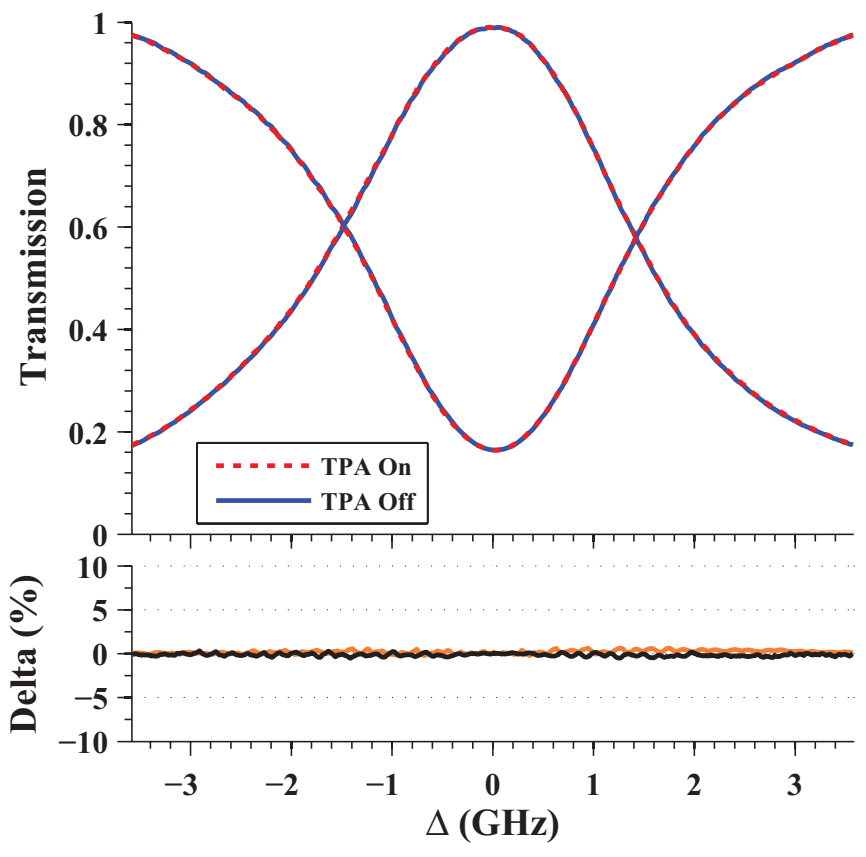

FIG. 5. (Color online) Control data demonstrating that no similar change is seen when $\mathrm{Rb}$ vapor is not present. Other controls are described in the text.

from two-photon resonance with the power levels, density, and frequency of the $780 \mathrm{~nm}$ free-space beam unchanged. In another, the position of the $780 \mathrm{~nm}$ beam was moved from the resonator to the waveguides to determine whether absorption before or after the resonator was an issue. All of our controls supported the conclusion that switching was due to TPA in the evanescent field of the microdisk.

Assuming symmetric fiber-coupling losses, the $1529 \mathrm{~nm}$ power in the input waveguide can be estimated to be roughly $130 \mathrm{nW}$ for the switching data shown in Fig. 4. The power in the free-space $780 \mathrm{~nm}$ beam was $30 \mathrm{~mW}$. The intensity of the 1529 beam in the resonator was $3.9 \mathrm{~kW} / \mathrm{cm}^{2}$ assuming a mode volume of $1.9 \times 10^{-11} \mathrm{~cm}^{3}\left[42(\lambda / n)^{3}\right]$. The intensity of the free-space beam $\left(940 \mathrm{~W} / \mathrm{cm}^{2}\right)$ would correspond to $26 \mathrm{nW}$ in the input waveguide.

The primary factor that affects switching contrast is the difference between the coupled cavity $Q$ with and without TPA. One way to improve the contrast of the switch is to increase the intrinsic quality factor allowing the total system $Q$ to be increased by decreasing the waveguide coupling strengths proportionally, leaving the coupling regime unchanged. Higher $Q \mathrm{Si}_{3} \mathrm{~N}_{4}$ devices have been demonstrated [25] using a variety of techniques and we are optimistic that we can improve the results presented here. We are also investigating the suitability of other types of microcavities for use in Zeno switching experiments, weighing factors such as intrinsic quality factor and mode volume with practical issues such as chip-scale integration and robustness.

The primary advantage of using an atomic vapor as compared to trapped atoms is the increased number of atoms in the mode and the practical advantages associated with not working at low temperature. The disadvantages of using atomic vapors are the effects of the atomic velocities and the buildup of the atoms on the surface of the device. The atomic velocities reduce the average time each atom spends in the mode, resulting in time-of-flight broadening [26]. In schemes such as the one demonstrated here, where the energies of the two atomic transitions are different, Doppler-free schemes are not effective and Doppler broadening dominates. A Dopplerfree scheme could be performed using the $5 S_{1 / 2} \rightarrow 5 P_{3 / 2} \rightarrow$ $5 D_{5 / 2}$ transition in $\mathrm{Rb}$, for example [16,17,27]. The atomic buildup on the surface of the device has been used as a tool in this experiment but it also results in slight degradations in the $Q$ that may become more important with lower-loss resonators. This coating on the surface of the device may also decrease the fraction of the mode that overlaps with the atomic vapor thereby reducing the effect of the atoms. Investigations are under way to determine the extent of these effects on switching performance, and preliminary results are encouraging [28].

\section{SUMMARY}

Here, we have presented an experimental demonstration of Zeno optical switching based on TPA. Using estimates based on the power of the free-space beam, all-optical switching with a control beam in the low nanowatt range seems attainable. Although the switching contrast is currently low, we believe the performance can be improved by addressing challenges related to device physics.

\section{ACKNOWLEDGMENTS}

We would like to acknowledge helpful discussions with Dave Clader. Funding was provided by the DARPA ZOE program (Contract No. W31P4Q-09-C-0566).
[1] K. Birnbaum, A. Boca, R. Miller, A. Boozer, T. Northup, and H. Kimble, Nature (London) 436, 87 (2005).

[2] S. M. Spillane, T. J. Kippenberg, K. J. Vahala, K. W. Goh, E. Wilcut, and H. J. Kimble, Phys. Rev. A 71, 013817 (2005).

[3] H. Mabuchi, Phys. Rev. A 80, 045802 (2009).

[4] D. Chang, A. Sørensen, E. Demler, and M. Lukin, Nat. Phys. 3, 807 (2007).

[5] R. Bose, D. Sridharan, H. Kim, G. S. Solomon, and E. Waks, Phys. Rev. Lett. 108, 227402 (2012).
[6] M. Pöllinger and A. Rauschenbeutel, Opt. Express 18, 17764 (2010).

[7] T. Tanabe, M. Notomi, S. Mitsugi, A. Shinya, and E. Kuramochi, Opt. Lett. 30, 2575 (2005).

[8] Q. Xu and M. Lipson, Opt. Lett. 31, 341 (2006).

[9] Q. Xu and M. Lipson, Opt. Express 15, 924 (2007).

[10] S. M. Spillane, G. S. Pati, K. Salit, M. Hall, P. Kumar, R. G. Beausoleil, and M. S. Shahriar, Phys. Rev. Lett. 100, 233602 (2008). 
[11] L. Stern, B. Desiatov, I. Goykhman, and U. Levy, arXiv:1204.0393.

[12] K. Salit, M. Salit, S. Krishnamurthy, Y. Wang, P. Kumar, and M. S. Shahriar, Opt. Express 19, 22874 (2011).

[13] S. M. Hendrickson, M. M. Lai, T. B. Pittman, and J. D. Franson, Phys. Rev. Lett. 105, 173602 (2010).

[14] W. Yang, D. Conkey, B. Wu, D. Yin, A. Hawkins, and H. Schmidt, Nat. Photonics 1, 331 (2007).

[15] L. Stern and U. Levy, Opt. Express 20, 28082 (2012).

[16] A. D. Slepkov, A. R. Bhagwat, V. Venkataraman, P. Londero, and A. L. Gaeta, Phys. Rev. A 81, 053825 (2010).

[17] B. C. Jacobs and J. D. Franson, Phys. Rev. A 79, 063830 (2009).

[18] Y. H. Wen, O. Kuzucu, M. Fridman, A. L. Gaeta, L.-W. Luo, and M. Lipson, Phys. Rev. Lett. 108, 223907 (2012).
[19] Y.-P. Huang, J. B. Altepeter, and P. Kumar, Phys. Rev. A 82, 063826 (2010).

[20] K. Kieu, L. Schneebeli, J. M. Hales, J. W. Perry, R. A. Norwood, and N. Peyghambarian, Opt. Express 19, 12532 (2011).

[21] B. Misra and E. C. G. Sudarshan, J. Math. Phys. 18, 756 (1977).

[22] Y. H. Wen, O. Kuzucu, T. Hou, M. Lipson, and A. L. Gaeta, Opt. Lett. 36, 1413 (2011).

[23] B. Little, H. Haus, J. Foresi, L. Kimerling, E. Ippen, and D. Ripin, IEEE Photonics Technol. Lett. 10, 816 (1998).

[24] E. R. Abraham and E. A. Cornell, Appl. Opt. 37, 1762 (1998).

[25] P. E. Barclay, K. Srinivasan, O. Painter, B. Lev, and H. Mabuchi, Appl. Phys. Lett. 89, 131108 (2006).

[26] S. Bagayev, V. Chebotayev, and E. Titov, Laser Phys. 4, 224 (1994).

[27] A. Olson, E. Carlson, and S. Mayer, Am. J. Phys. 74, 218 (2006).

[28] M. Lai, J. Franson, and T. Pittman, arXiv:1212.5189. 